\title{
ESTUDIO COMPARADO DEL RENDIMIENTO ACADÉMICO DEL ALUMNADO NATIVO E INMIGRANTE EN LA UNIÓN EUROPEA EN PISA 2015
}

\section{COMPARATIVE STUDY OF ACADEMIC PERFORMANCE OF NATIVE AND IMMIGRANT STUDENTS IN THE EUROPEAN UNION IN PISA 2015}

\author{
Adrián Neubauer Esteban
}

\begin{abstract}
Migration has turned as a new challenge for the European Union, as consequence of migration increasing in last years. Actually, quality is one of the most important objectives in educational agenda. However, this is not an easy question because governments need to find a balance between the quality and the universal access to education. Some researchers have been talked about this topic and they confirm the gap in the school performance between native and migrant students in the European Union. For that reason, this inquiry will compare school performance of native and migrant students in PISA 2015. It is important to highlight that we will differentiate between the immigrant of first and second generation to enrich the analysis. After we analyze the data about the competences in reading, mathematics and science, we will identify in which countries these differences are deeper or more superficial. Later, we will make some proposal to improve quality and equity in European's educational systems.
\end{abstract}

Key words: migrant; OECD; European Union; PISA; equality.

\section{RESUMEN}

Los sistemas educativos europeos se enfrentan a un nuevo reto como consecuencia de las numerosas migraciones que están teniendo lugar en los últimos años. El debate político, educativo y social se centra en cómo garantizar una educación de calidad a y a la vez se desarrolla a ciudadanía europea y se fomenta la cohesión social de todos los actores sociales. Ante este desafío, numerosas investigaciones han alertado sobre la gran diferencia existente entre el rendimiento académico del alumnado nativo y el alumnado inmigrante en la Unión Europea. Para conocer en mayor profundidad qué factores influyen en esta cuestión, vamos a comparar y analizar los datos obtenidos por las dos poblaciones en el informe PISA 2015. Es importante destacar que diferenciaremos entre la primera y la segunda generación de inmigrantes para enriquecer el análisis. Una vez analicemos los datos en la competencia lectora, la científica y la matemática, identificaremos en qué países estas diferencias son más profundas o superficiales. Más tarde, llevaremos a cabo una serie de propuestas de mejora en aras de una mayor equidad socioeducativa y primando el interés superior del menor.

Palabras clave: migrante; OCDE; Unión Europea; PISA; igualdad.

Fecha de recepción: 3 de julio de 2019.

Fecha de aceptación: 23 de septiembre de 2019. 


\section{INTRODUCCIÓN}

En los últimos años una palabra ha centrado el debate educativo: la calidad. Tradicionalmente se ha vinculado a obtener buenos resultados académicos y a tener éxito en la escuela (Monarca, 2012). Ante el deseo global de conocer qué sistemas educativos obtienen mejores resultados académicos emergió el informe PISA de la OCDE, arrojando luz sobre esta cuestión. Este instrumento de evaluación ha ganado protagonismo paulatinamente hasta ser una de las cuestiones candentes en educación (Pérez y Soto, 2011).

Además, el escenario político y social de la Unión Europea ha cambiado por completo en los últimos años como consecuencia de las numerosas migraciones que han tenido lugar dentro de las fronteras europeas. Esta nueva configuración social supone un fuerte desafío para la Unión Europea y para la OCDE (Rodríguez Izquierdo, 2010). Los sistemas educativos deben hacer frente a unas elevadas cotas de diversidad y multiculturalidad mientras garantizan la calidad de sus sistemas. La política educativa de la Unión Europea tiene una tarea más compleja todavía, garantizar la plena inclusión de las personas inmigrantes y favorecer la mayor cohesión social posible (Martínez-Usarralde, Lloret-Catalá y Céspedes, 2017).

De tal modo, tras ofrecer una visión general de la inmigración en la Unión Europea, en este trabajo vamos a describir las principales teorías y los factores que suponen una barrera para el éxito educativo de la población inmigrante. Después analizaremos los datos obtenidos por los Estados Miembro en la prueba de PISA 2015, comparando los resultados académicos de su población nativa y del alumnado inmigrante en las tres competencias: la científica, la lectora y la matemática. Del análisis podremos extraer las convergencias y las divergencias existentes entre los países con mayor diferencia en el rendimiento escolar entre su población nativa y la inmigrante, para finalizar con una serie de propuestas de mejora en base a los datos obtenidos.

\section{MARCO TEÓRICO}

\subsection{ANÁLISIS DE LA INMIGRACIÓN EN LA UNIÓN EUROPEA Y EL DERECHO A LA EDUCACIÓN}

La configuración sociopolítica y económica de la Unión Europea ha cambiado por completo desde el año 2015, momento en el que el número de solicitudes de asilo político se incrementaron de forma exponencial respecto a la tendencia habitual. Ese año el número de solicitudes aumentó hasta las 1.332.800, un número muy superior a las 627.000 que recibió la Unión Europea en 2014 (Comisión Europea, 2018a). Las causas de este hecho insólito en la Unión son variadas, aunque podemos encontrar un punto común en todas ellas: la violación sistemática de los derechos humanos en sus países de origen. Destaca el caso de Oriente medio, pues esta región geográfica se encuentra sumida en una Guerra Civil desde 2011 tras la irrupción de la Primavera Árabe y la convulsión social y política que causó en los países árabes, especialmente en Siria con Bashar AlAssad. Se calcula que esta guerra se ha cobrado más de 500.000 muertes y ha obligado 5,6 a millones de personas a emigrar de su país (ACNUR, 2018). En este contexto, no es de extrañar que las principales nacionalidades de estas personas sean la iraquí ( $7 \%$ del total de solicitudes de asilo) y la afgana (7\%) (Comisión Europea, 2018a).

Los principales países de destino para estas personas son Alemania (31\%), Italia (20\%), Francia (14\%), Grecia (9\%) y España (5\%) (Comisión Europea, 2018a). Es posible observar cómo estos países se sitúan en el sur de Europa, excepto Alemania, y son puntos de entrada al continente europeo, especialmente a través de la Ruta del Mediterráneo (UNICEF, 2017). Sin embargo, si 
observamos la Ruta de los Balcanes y la distribución de los solicitantes de asilo, sorprende no ver a Hungría y Polonia entre los primeros puestos de solicitudes de asilo. Si analizamos la situación política de estos países, podremos reconocer la ideología nacionalista y de ultraderecha actual de sus gobiernos. Esto implica que la política migratoria de estos países es muy dispar a la alemana, donde existe una actitud más favorable por parte del gobierno.

Tabla 1: Porcentaje de alumnado inmigrante en PISA 2015 en la Unión Europea

\begin{tabular}{|l|c|l|c|}
\hline ESTADO & PORCENTAJE & ESTADO & PORCENTAJE \\
\hline Alemania & 16,9 & Grecia & 10,8 \\
\hline Austria & 20,3 & Hungría & 2,7 \\
\hline Bélgica & 17,7 & Irlanda & 14,4 \\
\hline Bulgaria & 1,0 & Italia & 8,0 \\
\hline Chequia & 3,4 & Letonia & 5,0 \\
\hline Chipre & 11,3 & Lituania & 1,8 \\
\hline Croacia & 10,8 & Luxemburgo & 52,0 \\
\hline Dinamarca & 10,7 & Malta & 5,0 \\
\hline Eslovaquia & 1,2 & Países Bajos & 10,7 \\
\hline Eslovenia & 7,8 & Polonia & 0,3 \\
\hline España & 11,0 & Portugal & 7,3 \\
\hline Estonia & 10,0 & Reino Unido & 16,7 \\
\hline Finlandia & 4,0 & Rumanía & 0,4 \\
\hline Francia & 13,2 & Suecia & 17,4 \\
\hline
\end{tabular}

Fuente: elaboración propia a partir de OECD (2016). PISA 2015 Results (Volume I): Excellence and Equity in Education. OECD Publishing, Paris. doi: 10.1787/19963777

Además, si prestamos atención al número de solicitudes de los menores de edad, esta cuestión cobra gran interés, pues el modo en que la Unión Europea atienda y garantice los derechos humanos de estos menores influirá profundamente en su percepción y pertenencia a este organismo supranacional en el futuro. Los menores de edad representan cerca de un tercio del total de solicitudes (Comisión Europea, 2018a), por lo que garantizar sus derechos y libertades fundamentales acorde a la legislación internacional debe ocupar un papel central de la política de la Unión Europea.

Por otro lado, no podemos olvidar la multiculturalidad intrínseca que caracteriza a la Unión Europea dada su composición internacional. La Comisión (2018b) calcula que cerca de 22 millones de personas procedentes de terceros países viven en la Unión Europea, lo que supone el 4,2\% de su población total. Si a este dato le sumamos las migraciones internas que tienen lugar en el seno de los países europeos alcanzamos cifras realmente altas. En 2017, 16,9 millones de personas residían en un país diferente a su lugar de origen pese a permanecer dentro de la Unión Europea (Comisión Europea, 2018b). Estas cifras indican que la cuestión migratoria es un contenido que debe estar presente y orientar los programas educativos de los organismos internacionales, las autoridades nacionales y locales. De esta forma, la educación se sitúa a la cabeza de las medidas que debe adoptar la Unión Europea para promover y consolidar una ciudadanía europea. 


\subsection{LA VULNERABILIDAD ESCOLAR Y LABORAL DE LA POBLACIÓN INMIGRANTE: SUS PRINCIPALES FACTORES}

\subsubsection{TEORÍAS EXPLICATIVAS}

Rodríguez Izquierdo (2010) describe las principales teorías que explican las dificultades y las barreras que se encuentra la población inmigrante en el ámbito educativo y su menor éxito académico frente al alumnado de origen nativo. Las primeras teorías son "la teoría del déficit" y "la teoría de la diferencia". Ambas parten de la premisa de que el alumnado inmigrante carece de una base cultural previa que le permite triunfar en la escuela. Rodríguez Izquierdo (2010) señala tres consecuencias de adoptar esta concepción del alumnado inmigrante: 1) este alumnado está destinado a escolarizarse en programas compensatorios; 2) el alumno se enfrenta a la difícil decisión de elegir entre su cultura o adoptar la mayoritaria; 3) en último lugar, el docente tiene unas expectativas más bajas de este tipo de alumnado. Regueiro et al. (2018) ratifican esta última idea al señalar que los profesores creen que hacer deberes es una práctica más beneficiosa para el alumnado nacional que para los inmigrantes. Como bien es sabido las expectativas del docente influyen en el rendimiento académico del alumnado, lo que se conoce como el efecto Pigmalión (García Vargas, 2015). Por otro lado, "la teoría de la diferencia" es la respuesta a la teoría explicada anteriormente. Esta teoría permitió alcanzar mejores resultados al alumnado inmigrante a través de un lenguaje más directo, más claro y con menor complejidad (Rodríguez Izquierdo, 2010).

"La teoría de la discontinuidad cultural" tiene un gran valor pedagógico y sociológico, pues en ella se explican el fracaso escolar y el bajo rendimiento académico del alumnado inmigrante porque son educados en una cultura y en una lengua ajena a la suya, ya que son personas que reciben una educación diseñada y destinada a una población distinta a la suya: la clase media blanca (Rodríguez Izquierdo, 2010).

Por otro lado, "la teoría de la construcción social de la desventaja" se centra en la percepción de la población inmigrante acerca de su situación y de las barreras que le impiden progresar socialmente. Estos obstáculos se presentan como insuperables y les hacen sentir indefensos y vulnerables respecto a la cultura hegemónica. El éxito escolar es concebido como un "valor de la cultura blanca, con lo que entienden tener éxito como 'actuar como los blancos' (acting white)" (Rodríguez Izquierdo, 2010, p. 102). La aparente inmovilidad social que padecen estas minorías étnicas se traduce en un bajo rendimiento escolar, en un menor esfuerzo escolar y en una falta de motivación académica, al considerar que finalizar sus estudios no les brindará mejores oportunidades laborales ni sociales en el futuro.

En último lugar, "la teoría del capital social” es posiblemente la más compleja y holística de las que hemos presentado. Hay cuatro pilares fundamentales en esta teoría explicativa: la educación de las familias, las expectativas familiares sobre la educación de sus hijos, el compromiso familiar y el estilo parental. Esta teoría defiende que a mayores niveles en estas cuatro categorías el alumnado obtendrá mejores resultados escolares, siendo el nivel de estudios de la madre el más influyente de todos ellos (Calero, Choi y Waisgrais, 2009).

\subsubsection{OBSTÁCULOS EN EL RENDIMIENTO ACADÉMICO DEL ALUMNADO INMIGRANTE}

Después de haber descrito algunas teorías que explican el bajo rendimiento del alumnado inmigrante en los sistemas educativos formales, vamos a presentar los principales obstáculos a los que se enfrentan según la literatura académica: la titularidad del centro, el aprendizaje de la lengua de acogida, el bajo rendimiento escolar, el uso de la lengua materna en el hogar, el rechazo y la discriminación, la formación del profesorado, los factores socioeconómicos y el género. 
En primer lugar, el alumnado inmigrante se encuentra mayoritariamente agrupado en centros de titularidad pública (Zinovyeva, Felgueroso y Vázquez, 2009; Save the Children, 2018). En el caso de España, el 32\% del alumnado nacional está escolarizado en escuelas privadas y el 28\% en centros concertados (Save the Children, 2018). Esto supone que los centros públicos tienen entre el doble y el triple de alumnado inmigrante respecto a los centros concertados y privados. Estos datos coinciden con los obtenidos por Zinovyeva, Felgueroso y Vázquez (2009, p. 152), quienes afirman que los alumnos inmigrantes, "nacidos en el extranjero y cuyos padres también son extranjeros tienen cerca de un 50\% menos de probabilidad que los nativos de estudiar en escuelas no publicas". En esta misma línea de investigación, Cordero, Crespo y Pedraja (2013) alertan de que el alumnado inmigrante que obtiene resultados muy inferiores a los nativos se encuentra en centros públicos. Además, señalan que cuando la concentración del alumnado inmigrante supera el $20 \%$ los resultados académicos se reducen drásticamente.

Una de las grandes dificultades que se encuentra el alumnado inmigrante es el aprendizaje de la lengua de acogida. Los déficits lingüísticos tienen una fuerte influencia en la vida escolar del alumnado, especialmente en la enseñanza secundaria y la profesional (Etxeberría y Elosegui, 2010). La carencia lingǘstica repercute negativamente en todos los ámbitos vitales del alumno: le impide adquirir los conocimientos de la escuela, no se relaciona con los compañeros con fluidez, siente miedo y vergüenza por sus dificultades y termina por no expresarlas en busca de ayuda. Todo ello inevitablemente se refleja "en mayores dificultades para su integración social y laboral posterior" (Etxeberría y Elosegui, 2010, p. 242).

Uno de los factores, o la gran repercusión de esos factores, que más ha sido estudiada ha sido el bajo rendimiento académico del alumnado inmigrante, como se reflejó en los resultados del informe PISA de 2006 (Etxeberría y Elosegui, 2010) y de 2009 (Zinovyeva, Felgueroso y Vázquez, 2009). Etxeberría y Elosegui (2010) señalaron a Austria, Bélgica, Dinamarca, Francia, Alemania y Holanda como los países con mayores diferencias en la Unión Europea. Otra aportación del excelente trabajo de estos autores fue alertar sobre la gran disparidad entre el alumnado nativo y los de segunda generación. Resulta cuanto menos curiosa esta brecha en el rendimiento académico de ambos grupos de población porque los dos han sido escolarizados desde su nacimiento en el mismo sistema educativo. Sin embargo, Regueiro et al. (2018) hacen hincapié en que una de las razones por las que el alumnado nativo tiene mejores resultados es porque dedican más tiempo a los estudios y los realizan con mayor eficacia y calidad.

El bajo rendimiento del alumnado inmigrante se refleja en dos vías: el fracaso académico y el abandono escolar temprano (Martínez-Usarralde, Lloret-Catalá y Céspedes, 2017); y en las etapas educativas superiores, donde su presencia se reduce notablemente en comparación con la población nativa (Zinovyeva, Felgueroso y Vázquez, 2009). El informe PISA refleja fielmente las desventajas socioeducativas a las que se enfrenta el alumnado inmigrante, y nos alerta sobre la necesidad de desarrollar sistemas educativos más equitativos en aras de una mayor cohesión e igualdad social (Etxeberría y Elosegui, 2010; Martínez-Usarralde, Lloret-Catalá y Céspedes, 2017).

Por otro lado, otro factor de gran incidencia en el rendimiento escolar del alumnado inmigrante es que en su hogar se hable una lengua diferente a la del país de acogida. Esta práctica supone un gran escollo para el éxito educativo del alumnado (Etxeberría y Elosegui, 2010) y un reto para los sistemas educativos (Martínez-Usarralde, Lloret-Catalá y Céspedes, 2017).

Algunas de las barreras más preocupantes a las que se enfrenta el alumnado de origen inmigrante son el rechazo y la discriminación. Anteriormente hemos tratado la inequidad que existe en la 
distribución del alumnado inmigrante según la titularidad del centro escolar. Una de las razones por las que los Estados recurren a los centros gueto (Zinovyeva, Felgueroso y Vázquez, 2009) es por la negativa de la población nacional a que sus hijos compartan entornos de aprendizaje con el alumnado inmigrante (Martínez-Usarralde, Lloret-Catalá y Céspedes, 2017). Como apuntábamos previamente, los centros que cuentan con un porcentaje de inmigración mayor al 10\% y al 20\% influyen negativamente en los resultados académicos del alumnado (Calero, Choi y Waisgrais, 2009; Martínez-Usarralde, Lloret-Catalá y Céspedes, 2017). Sin embargo, las consecuencias de estos centros escolares son mucho profundas, pues perpetúan prejuicios y estereotipos racistas sobre esta población, lo que influye negativamente en la cohesión social y en la autoestima de alumnado inmigrante (Rodríguez Izquierdo, 2010; Martínez-Usarralde, Lloret-Catalá y Céspedes, 2017).

La formación del profesorado también se erige como otro factor influyente en el escaso rendimiento académico del alumnado inmigrante. Etxeberría, Karrera y Murua (2009) señalan las siguientes causas de este fenómeno:

1. El docente no tiene suficientes conocimientos acerca de la cultura, del país ni de la lengua de origen del alumnado inmigrante.

2. El profesorado no tiene las competencias adecuadas para adaptar o diseñar materiales y experiencias que mejoren el rendimiento del alumnado inmigrante.

3. Las bajas expectativas del profesorado sobre este alumnado.

4. El cuerpo docente dispone de poco tiempo, pocos recursos, escasa formación y apoyos para esta tarea. El autor destaca la precariedad de las condiciones para atender al alumnado de incorporación tardía.

Aunque también es importante apuntar que los docentes conocen y valoran positivamente recursos para atender a este alumnado, aunque los consideren insuficientes (Etxeberría, Karrera y Murua, 2009).

El entorno socioeconómico también tiene gran relevancia en esta cuestión. De hecho, Zinovyeva, Felgueroso y Vázquez (2009) señalan que es la principal causa de la diferencia en el rendimiento escolar entre el alumnado de origen nativo y el inmigrante. Estos autores apuntan a que el nivel de estudios de las familias es un factor relevante, pero Escarbajal, Sánchez y Guirao (2015) matizan que es el nivel de estudios materno el de mayor influencia. Cuanta menor formación académica tiene el entorno del alumno, sus posibilidades de fracasar escolarmente aumentan.

Para terminar, no podemos olvidar la barrera que supone el género para muchas estudiantes. El hecho de que algunas mujeres obtengan mejores resultados académicos que los hombres entra en conflicto directamente con el sistema y la estructura patriarcal de algunas culturas y familias (Rodríguez Izquierdo, 2010; Torrado, 2012).

\section{METODOLOGÍA}

A la hora de plantear esta investigación se han establecido los siguientes objetivos:

1. Identificar los países de la Unión Europea con menor y mayor brecha en el rendimiento escolar entre los menores inmigrantes y los nativos en las pruebas de PISA de 2015.

2. Describir la diferencia entre el rendimiento académico de los menores inmigrantes y los nativos en las pruebas PISA de 2015 en la Unión Europea.

3. Analizar las convergencias y las divergencias entre los Estados Miembro con menor y mayor brecha en el rendimiento escolar entre los menores migrantes y los nativos en las pruebas de PISA de 2015.

4. Realizar propuestas de mejora para garantizar una mayor equidad en las escuelas a partir de los tratados internacionales y supranacionales relacionados con el derecho a la educación. 
Para alcanzar dichos objetivos hemos diseñado un árbol de parámetros e indicadores en base a la prueba de PISA 2015. Por un lado, analizaremos el rendimiento del alumnado en las tres competencias que evalúa PISA: la competencia científica, la competencia lectora y la competencia matemática. Cada una de esas competencias será analizada a partir de dos grandes dimensiones: los resultados totales en la competencia y la diferencia entre la población nativa y migrante en esa competencia. La primera dimensión se describe comparando a la población nativa con la inmigrante. Sin embargo, el alumnado inmigrante se divide en tres subcategorías: el total, los de segunda generación (en adelante, SG) y los de primera generación (en adelante, PG). Este hecho se repite en la segunda dimensión, pero enriquecemos el análisis de los resultados atendiendo por un lado a la situación socioeconómica (en adelante, ISCED) y sin controlarla (en adelante, No ISCED). Así, es importante que hagamos una aclaración conceptual sobre los siguientes términos: nativo, primera generación y segunda generación. Para ello, partiremos de las definiciones de Etxeberría y Elosegui (2010, p. 244):

"Nativos: niños y niñas nacidos en el país de escolarización, con uno de los padres, al menos, nacido en ese país.

Primera generación o no nativos: niños nacidos en otro país, hijos de padres que también han nacido en otro país, distinto al de la escolarización.

Segunda generación: niños y niñas nacidos en el país de escolarización, pero con padres nacidos en otro país distinto".

Una vez obtengamos y analicemos los datos estableceremos dos grandes categorías de Estados: los que tienen mayores diferencias entre el alumnado inmigrante y el nativo, y los países con mayor equidad en esta cuestión. Más tarde, analizaremos en profundidad las similitudes y las diferencias intra e intermodelos para realizar propuestas de mejora a nivel europeo en el último apartado del trabajo.

\section{RESULTADOS}

\subsection{COMPETENCIA EN CIENCIAS}

Los resultados obtenidos por los estudiantes europeos en el informe PISA de 2015 en la competencia científica reflejan una gran brecha entre los estudiantes nativos y los de origen inmigrante. La nota media de los Estados Miembro en esta competencia fue de 494,2 puntos, mientras que el alumnado inmigrante obtuvo apenas 456,4. En términos cuantitativos la diferencia entre el alumnado local y el alumnado inmigrante fue de 39,7 puntos. Bien es cierto que si eliminamos la variable del contexto socioeconómico esta diferencia se reduce en diez puntos $(29,3)$, pero aún así, sigue existiendo una diferencia notable.

Dentro del alumnado inmigrante también es importante distinguir entre los de primera generación y los de segunda. Es posible apreciar como la diferencia entre el alumnado nacional y el de segunda generación es muy inferior $(31,9)$ respecto a los de primera generación $(48,1)$. Este hecho se repite tras eliminar la variable socioeconómica, reduciéndose la brecha para ambos grupos en once puntos. 
Tabla 2: Rendimiento académico de la población nativa e inmigrante en la competencia de ciencias en los Estados Miembro de la Unión Europea

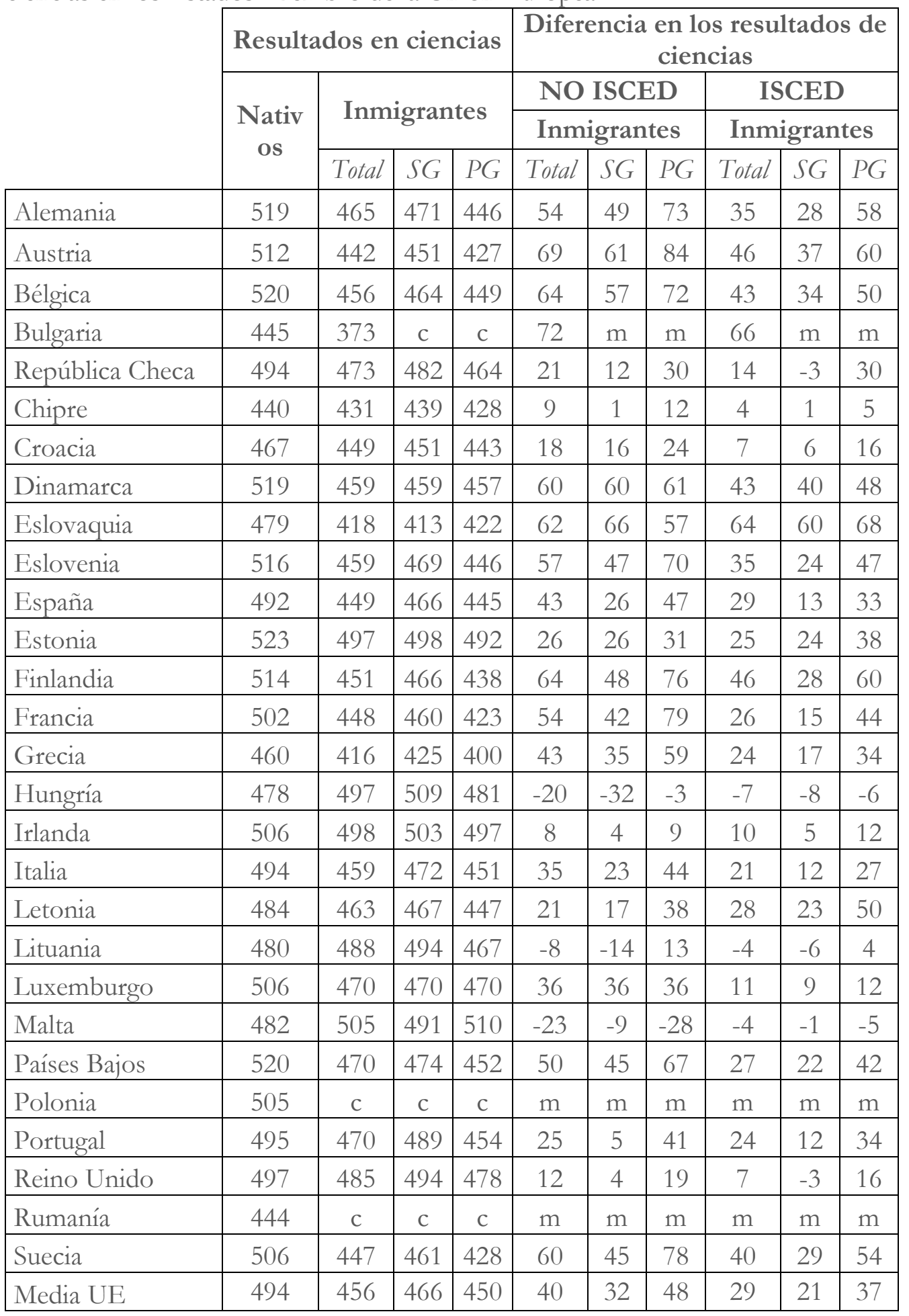

Fuente: elaboración propia a partir de OECD (2016). PISA 2015 Results (Volume I): Excellence and Equity in Education. OECD Publishing, Paris. doi: 10.1787/19963777 
Por otro lado, Finlandia (83), Bulgaria (74), Alemania (72), Eslovenia (71), Austria (70), Eslovaquia (70), Suecia (70) y Dinamarca (69) son los países donde existe mayor diferencia entre la población nacional y la de origen inmigrante. En el caso eslovaco la diferencia de rendimiento se incrementa en tres puntos si controlamos la variable socioeconómica.

Sin embargo, los países donde hay mayor equidad en los resultados académicos en esta competencia son: Portugal (16), Letonia (13), Lituania (8), Irlanda (5) y Chipre (1). En todos estos países, menos en Portugal, sucede algo muy llamativo, si controlamos la variable socioeconómica las diferencias entre la población inmigrante y nativa aumentan, especialmente en Letonia y Lituania. La mayor divergencia la establece Chipre, pues atendiendo a esta variable el alumnado inmigrante obtiene cuatro puntos más que el de origen nacional, mientras que si no tiene un punto menos.

En último lugar, llaman poderosamente la atención Hungría (-17) y Malta (-34). En ambos países el alumnado inmigrante obtiene resultados superiores al nacional. Estos dos países del este de Europa rompen completamente con la tendencia europea que hemos descrito anteriormente.

\subsection{COMPETENCIA LECTORA}

En términos generales, al analizar los resultados obtenidos en la competencia lectora por el alumnado europeo y el alumnado inmigrante seguimos encontrando esa diferencia entre ambos grupos. Por un lado, el alumnado nativo tiene unos resultados (494) significativamente superiores a los de origen inmigrante (456), pero si ahondamos en esta cuestión, podemos observar como la segunda generación de inmigrantes (466) tienen unos resultados superiores a los de primera generación (450) de nuevo.

Tabla 3: Rendimiento académico de la población nativa e inmigrante en la competencia de lectoescritura en los Estados Miembro de la Unión Europea

\begin{tabular}{|c|c|c|c|c|c|c|c|c|c|c|}
\hline & \multicolumn{4}{|c|}{$\begin{array}{l}\text { Resultados en } \\
\text { lectoescritura }\end{array}$} & \multicolumn{6}{|c|}{$\begin{array}{c}\text { Diferencia en los resultados de } \\
\text { lectoescritura }\end{array}$} \\
\hline & \multirow{3}{*}{ Nativos } & \multirow{2}{*}{\multicolumn{3}{|c|}{ Inmigrantes }} & \multirow{2}{*}{\multicolumn{3}{|c|}{$\begin{array}{c}\text { NO ISCED } \\
\text { Inmigrantes }\end{array}$}} & \multirow{2}{*}{\multicolumn{3}{|c|}{$\frac{\text { ISCED }}{\text { Inmigrantes }}$}} \\
\hline & & & & & & & & & & \\
\hline & & Total & $S G$ & $P G$ & Total & $S G$ & $P G$ & Total & $S G$ & $P G$ \\
\hline Alemania & 526 & 468 & 478 & 431 & 58 & 48 & 95 & 37 & 25 & 79 \\
\hline Austria & 499 & 435 & 448 & 413 & 64 & 51 & 85 & 38 & 25 & 58 \\
\hline Bélgica & 512 & 452 & 457 & 448 & 60 & 55 & 64 & 38 & 33 & 43 \\
\hline Bulgaria & 437 & 343 & $\mathrm{c}$ & $\mathrm{c}$ & 94 & $\mathrm{~m}$ & $\mathrm{~m}$ & 87 & $\mathrm{~m}$ & $\mathrm{~m}$ \\
\hline República Checa & 490 & 453 & 465 & 441 & 37 & 25 & 49 & 29 & 8 & 49 \\
\hline Chipre & 446 & 438 & 454 & 432 & 7 & -9 & 14 & 3 & -9 & 8 \\
\hline Croacia & 491 & 470 & 468 & 476 & 21 & 22 & 15 & 11 & 12 & 6 \\
\hline Dinamarca & 507 & 448 & 448 & 449 & 59 & 59 & 58 & 41 & 39 & 45 \\
\hline Eslovaquia & 458 & 370 & 386 & 354 & 88 & 72 & 104 & 90 & 64 & 116 \\
\hline Eslovenia & 511 & 459 & 478 & 434 & 51 & 33 & 77 & 28 & 9 & 52 \\
\hline España & 502 & 461 & 482 & 457 & 40 & 19 & 45 & 26 & 7 & 30 \\
\hline Estonia & 523 & 494 & 492 & 518 & 29 & 31 & 5 & 28 & 30 & 12 \\
\hline Finlandia & 531 & 448 & 484 & 419 & 83 & 47 & 112 & 64 & 26 & 96 \\
\hline Francia & 510 & 453 & 469 & 423 & 57 & 41 & 87 & 24 & 10 & 48 \\
\hline
\end{tabular}




\begin{tabular}{|l|c|c|c|c|c|c|c|c|c|c|} 
Grecia & 474 & 428 & 437 & 411 & 46 & 37 & 63 & 22 & 15 & 33 \\
\hline Hungría & 470 & 491 & 507 & 469 & -21 & -37 & 1 & -8 & -12 & -2 \\
\hline Irlanda & 525 & 511 & 519 & 509 & 14 & 5 & 16 & 16 & 6 & 19 \\
\hline Italia & 491 & 441 & 464 & 426 & 49 & 27 & 64 & 34 & 16 & 46 \\
\hline Letonia & 489 & 477 & 485 & 446 & 12 & 4 & 43 & 19 & 10 & 56 \\
\hline Lituania & 475 & 469 & 477 & 441 & 6 & -2 & 34 & 11 & 7 & 24 \\
\hline Luxemburgo & 503 & 465 & 467 & 461 & 38 & 36 & 42 & 10 & 6 & 15 \\
\hline Malta & 451 & 472 & 463 & 475 & -21 & -12 & -25 & -1 & -4 & 1 \\
\hline Países Bajos & 510 & 463 & 470 & 434 & 47 & 40 & 76 & 21 & 14 & 47 \\
\hline Polonia & 507 & $\mathrm{c}$ & $\mathrm{c}$ & $\mathrm{c}$ & $\mathrm{m}$ & $\mathrm{m}$ & $\mathrm{m}$ & $\mathrm{m}$ & $\mathrm{m}$ & $\mathrm{m}$ \\
\hline Portugal & 499 & 493 & 512 & 478 & 7 & -12 & 22 & 7 & -6 & 16 \\
\hline Reino Unido & 504 & 487 & 502 & 473 & 17 & 2 & 30 & 13 & -4 & 27 \\
\hline Rumanía & 434 & $\mathrm{c}$ & $\mathrm{c}$ & $\mathrm{c}$ & $\mathrm{m}$ & $\mathrm{m}$ & $\mathrm{m}$ & $\mathrm{m}$ & $\mathrm{m}$ & $\mathrm{m}$ \\
\hline Suecia & 514 & 453 & 477 & 423 & 60 & 37 & 90 & 40 & 21 & 64 \\
\hline Media UE & 492 & 455 & 472 & 446 & 39 & 25 & 51 & 28 & 14 & 40 \\
\hline
\end{tabular}

Fuente: elaboración propia a partir de OECD (2016). PISA 2015 Results (Volume I): Excellence and Equity in Education. OECD Publishing, Paris. doi: 10.1787/19963777

En la competencia lectora podemos encontrar a Austria, Dinamarca, Finlandia, Alemania, Eslovaquia, Suecia y Bulgaria a la cabeza de los países con mayores diferencias entre los dos grupos de estudio. De estos casos resulta especialmente alarmante el de Bulgaria, pues la diferencia del rendimiento académico entre el alumnado inmigrante y el nativo es de 94 puntos. Para más inri, si controlamos la variable socioeconómica podemos ver como esta diferencia apenas se reduce en siete puntos. Otros países que encabezan esta lista son Bélgica (60) y Francia (57), con una diferencia en el rendimiento muy similar.

Hay tres países que establecen una divergencia respecto al resto de Estados Miembro: Eslovaquia, Irlanda y Lituania. En ellos la diferencia en el rendimiento académico aumenta si controlamos la variable socioeconómica. El aumento es de apenas dos (Eslovaquia e Irlanda) y cinco puntos (Lituania), pero es un fenómeno que convendría estudiar con mayor detalle en futuras investigaciones, especialmente en el caso eslovaco, pues su diferencia es de las más altas en esta competencia.

Irlanda (14), Chipre (7) y Lituania (6) se mantienen como los países de referencia en equidad en los resultados académicos entre el alumnado inmigrante y el nativo. En esta competencia también destacan Reino Unido (17) y Croacia (21) como los países que tienen una menor brecha entre el rendimiento escolar de su alumnado inmigrante y nativo. Para terminar, el caso más esperanzador es el chipriota. La diferencia es de apenas tres puntos cuando atendemos a la variable socioeconómica, lo que en términos generales podríamos reconocer como un sistema de éxito en la integración del alumnado inmigrante.

\subsection{COMPETENCIA MATEMÁTICA}

La competencia matemática reduce ligeramente la diferencia en el rendimiento académico entre el alumnado inmigrante y el alumnado nativo. Sin embargo, estas diferencias siguen siendo profundas, pues el alumnado inmigrante se sitúa 34 puntos por debajo respecto al de origen nacional. En esta 
competencia también se aprecia una diferencia significativa entre el alumnado de origen inmigrante, obteniendo los de segunda generación 18 puntos más que los de primera generación.

Tabla 4: Rendimiento académico de la población nativa e inmigrante en la competencia matemática en los Estados Miembro de la Unión Europea

\begin{tabular}{|c|c|c|c|c|c|c|c|c|c|c|}
\hline & \multicolumn{4}{|c|}{$\begin{array}{l}\text { Resultados en } \\
\text { matemáticas }\end{array}$} & \multicolumn{6}{|c|}{$\begin{array}{c}\text { Diferencia en los resultados de } \\
\text { matemáticas }\end{array}$} \\
\hline & \multirow{3}{*}{ Nativos } & \multirow{2}{*}{\multicolumn{3}{|c|}{ Inmigrantes }} & \multirow{2}{*}{\multicolumn{3}{|c|}{$\begin{array}{l}\text { NO ISCED } \\
\text { Inmigrantes }\end{array}$}} & \multirow{2}{*}{\multicolumn{3}{|c|}{$\frac{\text { ISCED }}{\text { Inmigrantes }}$}} \\
\hline & & & & & & & & & & \\
\hline & & Total & $S G$ & $P G$ & Total & $S G$ & $P G$ & Total & $S G$ & $P G$ \\
\hline Alemania & 519 & 465 & 471 & 446 & 54 & 49 & 73 & 35 & 28 & 58 \\
\hline Austria & 512 & 442 & 451 & 427 & 69 & 61 & 84 & 46 & 37 & 60 \\
\hline Bélgica & 520 & 456 & 464 & 449 & 64 & 57 & 72 & 43 & 34 & 50 \\
\hline Bulgaria & 445 & 373 & $\mathrm{c}$ & $\mathrm{c}$ & 72 & $\mathrm{~m}$ & $\mathrm{~m}$ & 66 & $\mathrm{~m}$ & $\mathrm{~m}$ \\
\hline Chequia & 494 & 473 & 482 & 464 & 21 & 12 & 30 & 14 & -3 & 30 \\
\hline Chipre & 440 & 431 & 439 & 428 & 9 & 1 & 12 & 4 & 1 & 5 \\
\hline Croacia & 467 & 449 & 451 & 443 & 18 & 16 & 24 & 7 & 6 & 16 \\
\hline Dinamarca & 519 & 459 & 459 & 457 & 60 & 60 & 61 & 43 & 40 & 48 \\
\hline Eslovaquia & 479 & 418 & 413 & 422 & 62 & 66 & 57 & 64 & 60 & 68 \\
\hline Eslovenia & 516 & 459 & 469 & 446 & 57 & 47 & 70 & 35 & 24 & 47 \\
\hline España & 492 & 449 & 466 & 445 & 43 & 26 & 47 & 29 & 13 & 33 \\
\hline Estonia & 523 & 497 & 498 & 492 & 26 & 26 & 31 & 25 & 24 & 38 \\
\hline Finlandia & 514 & 451 & 466 & 438 & 64 & 48 & 76 & 46 & 28 & 60 \\
\hline Francia & 502 & 448 & 460 & 423 & 54 & 42 & 79 & 26 & 15 & 44 \\
\hline Grecia & 460 & 416 & 425 & 400 & 43 & 35 & 59 & 24 & 17 & 34 \\
\hline Hungría & 478 & 497 & 509 & 481 & -20 & -32 & -3 & -7 & -8 & -6 \\
\hline Irlanda & 506 & 498 & 503 & 497 & 8 & 4 & 9 & 10 & 5 & 12 \\
\hline Italia & 494 & 459 & 472 & 451 & 35 & 23 & 44 & 21 & 12 & 27 \\
\hline Letonia & 484 & 463 & 467 & 447 & 21 & 17 & 38 & 28 & 23 & 50 \\
\hline Lituania & 480 & 488 & 494 & 467 & -8 & -14 & 13 & -4 & -6 & 4 \\
\hline Luxemburgo & 506 & 470 & 470 & 470 & 36 & 36 & 36 & 11 & 9 & 12 \\
\hline Malta & 482 & 505 & 491 & 510 & -23 & -9 & -28 & -4 & -1 & -5 \\
\hline Países Bajos & 520 & 470 & 474 & 452 & 50 & 45 & 67 & 27 & 22 & 42 \\
\hline Polonia & 505 & $\mathrm{c}$ & $\mathrm{c}$ & $\mathrm{c}$ & $\mathrm{m}$ & $\mathrm{m}$ & $\mathrm{m}$ & $\mathrm{m}$ & $\mathrm{m}$ & $\mathrm{m}$ \\
\hline Portugal & 495 & 470 & 489 & 454 & 25 & 5 & 41 & 24 & 12 & 34 \\
\hline Reino Unido & 497 & 485 & 494 & 478 & 12 & 4 & 19 & 7 & -3 & 16 \\
\hline Rumanía & 444 & $\mathrm{c}$ & $\mathrm{c}$ & $\mathrm{c}$ & $\mathrm{m}$ & $\mathrm{m}$ & $\mathrm{m}$ & $\mathrm{m}$ & $\mathrm{m}$ & $\mathrm{m}$ \\
\hline Suecia & 506 & 447 & 461 & 428 & 60 & 45 & 78 & 40 & 29 & 54 \\
\hline Media UE & 493 & 459 & 470 & 452 & 35 & 27 & 44 & 26 & 17 & 33 \\
\hline
\end{tabular}

Fuente: elaboración propia a partir de OECD (2016). PISA 2015 Results (Volume I): Excellence and Equity in Education. OECD Publishing, Paris. doi: 10.1787/19963777 
Los países que obtienen mayores diferencias en el rendimiento matemático son los mismos que hemos nombrado en la competencia científica y la competencia lectora. Bulgaria es el país donde estas diferencias se hacen más significativas, pues su alumnado nativo obtuvo una nota de 445 puntos, mientras que el inmigrante apenas 373. Es interesante observar cómo una vez más en este país la diferencia tan sólo se reduce en seis puntos al controlar la variable socioeconómica.

Los países del norte de Europa presentan diferencias muy similares entre sus estudiantes. Austria (69), Bélgica (64), Finlandia (64), Eslovaquia (62), Dinamarca (60) y Suecia (60) son países cuyas diferencias internas superan los sesenta puntos. Mención especial merecen Eslovaquia e Irlanda, pues son los dos únicos países en los que controlando la variable socioeconómica las diferencias aumentan ligeramente. Es llamativo observar cómo dos países tan divergentes en equidad de resultados coinciden en esta cuestión.

Datos más positivos arrojan Croacia, Reino Unido, Chipre, Irlanda y Lituania, pues la diferencia de los resultados entre el alumnado nativo y el inmigrante no supera la veintena de puntos en ninguno de los casos. En estos países se aprecia una similitud clara, apenas hay diferencia en los resultados controlando la variable socioeconómica. En todos los casos se reduce ligeramente la diferencia a excepción de Irlanda como apuntábamos anteriormente.

Para terminar, hay tres países que divergen del resto de países europeos: Malta, Hungría y Lituania. En ellos el alumnado inmigrante obtiene mejores resultados en matemáticas que los de origen nativo. En Malta el alumnado inmigrante tiene un rendimiento superior en 23 puntos y tras controlar la variable socioeconómica esta brecha se reduce a 4 puntos. El caso húngaro es muy similar al de Malta, pues mientras el alumnado nativo obtiene 478 puntos, los de origen inmigrante obtienen 497 puntos. Si vamos más allá, el alumnado inmigrante de segunda generación tiene un rendimiento muy superior, alcanzando los 505 puntos. En último lugar, el país que obtiene mejores resultados en equidad es Lituania. La diferencia entre estos dos grupos de estudiantes es de apenas ocho puntos, en favor de los de origen inmigrante. Controlando la variable socioeconómica la diferencia se reduce a cuatro puntos, al igual que sucede en el país chipriota.

\subsection{ELEMENTOS COMUNES Y DIVERGENCIAS}

Una vez detallados los resultados obtenidos en la investigación, vamos a analizar con detalle qué elementos comunes y qué diferencias hemos encontrado en los países con mayores y menores divergencias en el rendimiento escolar del alumnado nativo e inmigrante en PISA 2015.

La primera convergencia que hemos observado en los países con diferencias más significativas entre el alumnado inmigrante y el nativo es su localización geográfica. Estos países se encuentran en la zona norte de Europa, a excepción de Bulgaria situada al sureste. Tradicionalmente, en la literatura académica se ha reconocido el gran potencial académico de estos países europeos destacando Finlandia por encima de todos (Enkvist, 2010; Gripenberg y Lizarte, 2012; Sahlberg, 2015). Estas afirmaciones enmarcan perfectamente la segunda conclusión que hemos extraído del análisis. Los países que obtienen mayores diferencias entre la población nativa y el alumnado inmigrante tienen unos resultados superiores a 500 puntos en todas las competencias. Los únicos países que no siguen este modelo son Bulgaria y Eslovaquia, que tienen puntuaciones inferiores en todas las pruebas realizadas, siendo Bulgaria el país con los resultados más bajos. Por el contrario, los países más equitativos obtienen resultados inferiores a 500 puntos en todas las competencias, a excepción de Portugal, Reino Unido e Irlanda. Este último país alcanza puntuaciones mucho mayores que los países con más equidad, pues supera los 500 puntos en la prueba de ciencias (505), de lectura (525) y de matemáticas (506). Sin embargo, Portugal sólo supera los 500 puntos en la prueba de ciencias, lo mismo que le sucede a Reino Unido en la competencia lectora. 
Bulgaria y Eslovaquia son la máxima expresión de la tercera conclusión a la que llegamos acerca de los países con mayor desigualdad: tienen porcentajes de alumnado inmigrante muy bajos. Entre los cinco países con más diferencia en la competencia científica Alemania es el único país con una tasa elevada de alumnado inmigrante (17\%), pues Finlandia (4\%), Eslovenia (8\%) y especialmente Bulgaria $(1 \%)$ y Eslovaquia $(1 \%)$ tienen porcentajes muy bajos. En la competencia lectora encontramos a Austria $(20 \%)$ y Suecia $(17 \%)$ entre las cinco con mayores diferencias, pero los porcentajes de alumnado inmigrante son muy superiores en comparación a Bulgaria, Eslovaquia y Finlandia. Lo mismo sucede en la competencia matemática con Austria y Bélgica (18\%). En las tres competencias podemos vislumbrar como a partir de los cinco países más desiguales los porcentajes de alumnado inmigrante aumentan considerablemente. Sin embargo, en los países con mayor equidad encontramos a Letonia $(5 \%)$ y Lituania $(2 \%)$ con porcentajes muy bajos de alumnado inmigrante y a países como Reino Unido (17\%) o Irlanda (14\%) cuya proporción de este grupo de alumnos es más elevada. En términos generales podríamos afirmar que la diferencia del porcentaje de alumnado inmigrante entre los países más equitativos es inferior a aquellos países que tienen diferencias más altas en el rendimiento académico entre estos grupos de estudiantes.

La cuarta conclusión atiende a las diferencias que existen entre el alumnado inmigrante de primera y de segunda generación. En los países con mayor inequidad entre el alumnado nacional y el inmigrante aumenta considerablemente la desventaja académica del alumnado de primera generación respecto a los de segunda generación. Analizando la tabla observamos como las diferencias entre ambas generaciones son mucho mayores en los países con cotas de desigualdad más altas. Esto se refleja claramente en el promedio de las tres competencias comparando las diferencias en los resultados de ambas generaciones en estos dos modelos de países.

Tabla 5: Media de las diferencias en el rendimiento académico entre el alumnado inmigrante y de nativo de los países con mayores y menores diferencias de la UE

\begin{tabular}{|c|c|c|c|c|c|}
\hline & \multirow{2}{*}{\begin{tabular}{|l|}
$\begin{array}{l}\text { Países con más } \\
\text { diferencias }\end{array}$ \\
63 \\
\end{tabular}} & \multirow{2}{*}{\begin{tabular}{|l|}
$\begin{array}{l}\text { Países con menores } \\
\text { diferencias }\end{array}$ \\
0 \\
\end{tabular}} & \multirow{2}{*}{$\begin{array}{l}\begin{array}{l}\text { Diferencia entre } \\
\text { países }\end{array} \\
63 \\
\end{array}$} \\
\hline \multirow{4}{*}{ Ciencias } & \multirow{2}{*}{ NO ISCED } & $S G$ & & & \\
\hline & & $P G$ & 85 & 21 & 64 \\
\hline & \multirow{2}{*}{ ISCED } & $S G$ & 43 & 5 & 38 \\
\hline & & $P G$ & 69 & 19 & 50 \\
\hline \multirow{4}{*}{ Lectura } & \multirow{2}{*}{ NO ISCED } & $S G$ & 51 & 4 & 47 \\
\hline & & $P G$ & 87 & 22 & 65 \\
\hline & \multirow{2}{*}{ ISCED } & $S G$ & 30 & 3 & 27 \\
\hline & & $P G$ & 69 & 17 & 52 \\
\hline \multirow{4}{*}{ Matemáticas } & \multirow{2}{*}{ NO ISCED } & $S G$ & 53 & 2 & 51 \\
\hline & & $P G$ & 72 & 15 & 57 \\
\hline & \multirow{2}{*}{ ISCED } & $S G$ & 30 & 1 & 29 \\
\hline & & $P G$ & 69 & 11 & 58 \\
\hline \multirow{4}{*}{$\begin{array}{l}\text { Media de las tres } \\
\text { competencias }\end{array}$} & \multirow{2}{*}{ NO ISCED } & $S G$ & 56 & 2 & 54 \\
\hline & & $P G$ & 81 & 19 & 62 \\
\hline & \multirow{2}{*}{ ISCED } & $S G$ & 34 & 3 & 31 \\
\hline & & $P G$ & 69 & 16 & 53 \\
\hline
\end{tabular}

Fuente: elaboración propia a partir de OECD (2016). PISA 2015 Results (Volume I): Excellence and Equity in Education. OECD Publishing, Paris. doi: 10.1787/19963777 
Para terminar, la quinta conclusión emana directamente del último apartado de la tabla: la influencia del nivel socioeconómico. En los países con unos resultados más desiguales podemos observar como la diferencia entre ambas generaciones sin atender a la variable socioeconómica se sitúa en los 25 puntos, mientras que en los países más equitativos es de 17 puntos. Sin embargo, en estos últimos países cuando controlamos la variable socioeconómica la distancia entre ambas generaciones se reduce hasta los 13 puntos. Por el contrario, en los países con mayor desigualdad en el rendimiento académico aumenta hasta los 35 puntos. Así, podemos concluir que la variable socioeconómica es más influyente en los países con más desigualdad en el rendimiento entre el alumnado inmigrante y el nativo que en los de mayor igualdad.

No obstante, este análisis debe tener un análisis interpretativo más sistémico, que enriquezca y dote de una mayor significatividad a los datos mostrados anteriormente. Para ello, PISA nos ofrece tres indicadores óptimos, como son: el porcentaje de alumnado resiliente, la segregación escolar y los recursos que tienen las escuelas.

En el primer indicador es posible identificar cómo un número sensible de Estados con grandes diferencias en el rendimiento académico también destacan negativamente en este apartado. Finlandia (26,5), Austria (13,8), Dinamarca (13,8), Alemania (11,3), Eslovenia $(10,5)$ y Suecia $(10,1)$ se encuentran entre los países con diferencias significativas más considerable entre el porcentaje de alumnado resiliente entre los estudiantes nativos e inmigrantes. Sin embargo, en este indicador encontramos por primera vez a España $(14,6)$, Bélgica $(12,5)$, Portugal $(11,6)$ y Croacia $(7,1)$, países que hasta la fecha habían destacado por la paridad de resultados entre las dos poblaciones de estudiantes, especialmente Portugal y Croacia.

Tabla 6: Alumnado resiliente en los Estados Miembro de la Unión Europea.

\begin{tabular}{|l|c|c|c|c|}
\cline { 2 - 5 } \multicolumn{1}{c|}{} & Total & Nativos & Inmigrantes & $\begin{array}{c}\text { Diferencia entre nativos e } \\
\text { inmigrantes }\end{array}$ \\
\hline Alemania & 33,5 & 36,7 & 25,4 & 11,3 \\
\hline Austria & 25,9 & 31,3 & 17,5 & 13,8 \\
\hline Bélgica & 27,2 & 31,1 & 18,7 & 12,5 \\
\hline Bulgaria & 13,6 & 14,1 & $\mathrm{~m}$ & $\mathrm{~m}$ \\
\hline Chequia & 24,9 & 25,0 & 29,2 & $-4,2$ \\
\hline Chipre & 10,1 & 10,4 & 9,6 & 0,8 \\
\hline Croacia & 24,4 & 25,8 & 18,7 & 7,1 \\
\hline Dinamarca & 27,5 & 30,3 & 16,7 & 13,6 \\
\hline Eslovaquia & 17,5 & 18,2 & $\mathrm{~m}$ & $\mathrm{~m}$ \\
\hline Eslovenia & 34,6 & 36,6 & 26,1 & 10,5 \\
\hline España & 39,2 & 42,0 & 27,4 & 14,6 \\
\hline Estonia & 48,3 & 48,9 & 46,4 & 2,5 \\
\hline Finlandia & 42,8 & 44,9 & 18,4 & 26,5 \\
\hline Francia & 26,6 & 27,6 & 25,7 & 1,9 \\
\hline Grecia & 18,1 & 19,3 & 15,1 & 4,2 \\
\hline Hungría & 19,3 & 19,2 & $\mathrm{~m}$ & $\mathrm{~m}$ \\
\hline Irlanda & 29,6 & 28,9 & 37,4 & $-8,5$ \\
\hline Italia & 26,6 & 27,0 & 23,7 & 3,3 \\
\hline
\end{tabular}




\begin{tabular}{|l|c|c|c|c|} 
Letonia & 35,2 & 35,8 & 23,9 & 11,9 \\
\hline Lituania & 23,1 & 23,4 & 18,6 & 4,8 \\
\hline Luxemburgo & 20,7 & 22,8 & 20,4 & 2,5 \\
\hline Malta & 21,8 & 22,8 & $\mathrm{~m}$ & $\mathrm{~m}$ \\
\hline Países Bajos & 30,7 & 31,9 & 27,6 & 4,3 \\
\hline Polonia & 34,6 & 35,0 & $\mathrm{~m}$ & $\mathrm{~m}$ \\
\hline Portugal & 38,1 & 39,0 & 27,4 & 11,6 \\
\hline Reino Unido & 35,4 & 36,8 & 33,7 & 3,1 \\
\hline Rumanía & 11,3 & 11,1 & $\mathrm{~m}$ & $\mathrm{~m}$ \\
\hline Suecia & 24,7 & 27,9 & 17,8 & 10,1 \\
\hline Media UE & 27,3 & 28,7 & 23,9 & 7,2 \\
\hline
\end{tabular}

Fuente: elaboración propia a partir de OECD (2016). PISA 2015 Results (Volume I): Excellence and Equity in Education. OECD Publishing, Paris. doi: 10.1787/19963777

La segregación escolar ha sido rigurosa y vastamente reconocida como un elemento que influye negativamente en el rendimiento académico del alumnado (Calero, Choi y Waisgrais, 2009; Zinovyeva, Felgueroso y Vázquez, 2009; Cordero, Crespo y Pedraja, 2013; Save the Children, 2018). En primer lugar, llama poderosamente la atención cómo el informe PISA no ofrece esta información sobre 10 Estados Miembro de la Unión Europea (Bulgaria, Chequia, Eslovaquia, Finlandia, Hungría, Letonia, Lituania, Malta, Polonia y Rumanía). Por otro lado, analizando detenidamente la segregación escolar de la Unión Europea no podemos afirmar que la diferencia de rendimiento entre el alumnado inmigrante y nativo sea debida a este fenómeno. En algunos casos es posible identificar una convergencia entre estos indicadores como en Austria, Alemania, Suecia y Eslovenia, sin embargo, Reino Unido, Luxemburgo e Irlanda tienen unas de las mayores tasas de segregación escolar en toda la Unión y, sin embargo, estas diferencias en el rendimiento escolar no son tan acentuadas.

Tabla 7: Segregación escolar en 18 Estados Miembro de la Unión Europea.

\begin{tabular}{|l|c|c|c|}
\cline { 2 - 4 } \multicolumn{1}{c|}{} & $\begin{array}{c}\text { Índice de } \\
\text { concentración de } \\
\text { alumnado inmigrante } \\
\text { actual }\end{array}$ & $\begin{array}{c}\text { Índice máximo de la } \\
\text { concentración portencial de } \\
\text { alumnado inmigrante }\end{array}$ & Diferencia \\
\hline Alemania & 13,4 & 31,2 & 17,8 \\
\hline Austria & 15,8 & 38,6 & 22,9 \\
\hline Bélgica & 15,0 & 33,4 & 18,4 \\
\hline Chipre & 9,8 & 23,6 & 13,8 \\
\hline Croacia & 6,8 & 21,4 & 14,7 \\
\hline Dinamarca & 11,3 & 26,2 & 14,9 \\
\hline Eslovenia & 7,0 & 26,0 & 19,0 \\
\hline España & 9,6 & 22,5 & 12,9 \\
\hline Estonia & 9,8 & 19,6 & 12,8 \\
\hline Francia & 11,8 & 24,4 & 14,3 \\
\hline Grecia & 8,7 & 23,1 & 19,1 \\
\hline Irlanda & 8,7 & 27,9 & \\
\hline
\end{tabular}




\begin{tabular}{|l|c|c|c|} 
Italia & 6,5 & 28,2 & 21,7 \\
\hline Luxemburgo & 17,4 & 49,4 & 32,0 \\
\hline Países Bajos & 10,2 & 22,6 & 12,4 \\
\hline Portugal & 6,3 & 18,8 & 12,5 \\
\hline Reino Unido & 15,7 & 49,9 & 34,2 \\
\hline Suecia & 13,8 & 30,1 & 16,3 \\
\hline
\end{tabular}

Fuente: elaboración propia a partir de OECD (2016). PISA 2015 Results (Volume I): Excellence and Equity in Education. OECD Publishing, Paris. doi: 10.1787/19963777

En última instancia, PISA hace una diferenciación entre los recursos materiales y los recursos humanos, o docentes, de los que disponen las escuelas con baja y alta concentración de alumnado inmigrante. En cuanto a los recursos materiales tan sólo Italia y Estonia carecen significativamente de los recursos necesarios para las escuelas con alta concentración de población inmigrante. Mientras tanto en Luxemburgo, España, Alemania y Chipre encontramos una correlación positiva y significativa en estos centros educativos. Estos dos últimos países son casos contrarios pese a destinar un número importante de recursos materiales a estas escuelas, pues el país teutón padece una diferencia considerable entre el rendimiento de estas dos poblaciones de estudiantes, mientras que Chipre se caracteriza por una fuerte similitud entre ambas. Esto se repite en los recursos humanos y docentes entre Chipre, Croacia, Austria y Suecia, donde los países del norte de Europa, pese a disponer de más recursos obtienen resultados más desiguales entre los estudiantes inmigrantes y nativos. Además, Estonia y Luxemburgo carecen de los recursos humanos oportunos para atender a este alumnado, mientras que en España hay una sobredotación de personal docente destinada a satisfacer las necesidades y demandas del alumnado inmigrante.

Tabla 8: Escasez de recursos materiales y humanos en 18 Estados Miembro de la Unión Europea.

\begin{tabular}{|c|c|c|c|c|c|c|c|c|}
\hline & \multicolumn{5}{|c|}{ Índice de escasez de material educativo } & \multicolumn{3}{|c|}{ Indice de escasez. de recursos humanos } \\
\hline & $\begin{array}{l}\text { Todas } \\
\text { las } \\
\text { escuelas }\end{array}$ & $\begin{array}{c}\text { Baja } \\
\text { concentración } \\
\text { de } \\
\text { inmigrantes }\end{array}$ & $\begin{array}{c}\text { Alta } \\
\text { concentración } \\
\text { de } \\
\text { inmigrantes }\end{array}$ & Diferencia & $\begin{array}{l}\text { Todas } \\
\text { las } \\
\text { escuelas }\end{array}$ & $\begin{array}{c}\text { Baja } \\
\text { concentración } \\
\text { de } \\
\text { inmigrantes }\end{array}$ & $\begin{array}{c}\text { Alta } \\
\text { concentración } \\
\text { de } \\
\text { inmigrantes }\end{array}$ & Diferencia \\
\hline Alemania & 0,06 & $-0,08$ & 0,20 & 0,27 & 0,41 & 0,34 & 0,48 & 0,14 \\
\hline Austria & $-0,27$ & $-0,27$ & $-0,26$ & 0,01 & 0,18 & 0,00 & 0,36 & 0,36 \\
\hline Bélgica & 0,11 & 0,16 & 0,07 & $-0,08$ & 0,23 & 0,21 & 0,26 & 0,06 \\
\hline Chipre & $-0,06$ & $-0,28$ & 0,16 & 0,44 & 0,06 & $-0,13$ & 0,27 & 0,40 \\
\hline Croacia & 0,87 & 0,77 & 0,98 & 0,21 & $-0,02$ & $-0,25$ & 0,21 & 0,46 \\
\hline Dinamarca & $-0,21$ & $-0,23$ & $-0,17$ & 0,06 & $-0,70$ & $-0,71$ & $-0,68$ & 0,04 \\
\hline Eslovenia & $-0,30$ & $-0,30$ & $-0,30$ & 0,00 & $-0,52$ & $-0,56$ & $-0,47$ & 0,09 \\
\hline España & 0,23 & $-0,06$ & 0,50 & 0,56 & 0,27 & $-0,03$ & 0,55 & 0,58 \\
\hline Estonia & 0,05 & 0,21 & $-0,12$ & $-0,33$ & 0,07 & 0,21 & $-0,07$ & $-0,28$ \\
\hline Francia & $-0,17$ & $-0,19$ & $-0,14$ & 0,05 & 0,17 & 0,18 & 0,16 & $-0,02$ \\
\hline
\end{tabular}




\begin{tabular}{|l|c|c|c|c|c|c|c|c|} 
Grecia & 0,39 & 0,38 & 0,40 & 0,02 & 0,61 & 0,53 & 0,68 & 0,15 \\
\hline Irlanda & 0,25 & 0,41 & 0,10 & $-0,32$ & 0,12 & 0,17 & 0,08 & $-0,09$ \\
\hline Italia & 0,56 & 0,75 & 0,36 & $-\mathbf{0 , 3 9}$ & 0,35 & 0,35 & 0,35 & $-0,01$ \\
\hline Luxemburgo & $-0,16$ & $-0,19$ & $-0,13$ & $\mathbf{0 , 0 5}$ & 0,39 & 0,53 & 0,27 & $-\mathbf{0 , 2 6}$ \\
\hline Países Bajos & $-0,20$ & $-0,32$ & $-0,08$ & 0,24 & 0,01 & $-0,05$ & 0,08 & 0,13 \\
\hline Portugal & 0,11 & 0,02 & 0,17 & 0,16 & 0,93 & 0,82 & 1,01 & 0,19 \\
\hline Reino Unido & 0,04 & 0,07 & 0,02 & $-0,05$ & $-0,12$ & $-0,24$ & $-0,02$ & 0,22 \\
\hline Suecia & $-0,28$ & $-0,32$ & $-0,24$ & 0,09 & 0,35 & 0,20 & 0,49 & $\mathbf{0 , 2 9}$ \\
\hline
\end{tabular}

*Nota: En los números resaltados en negrita existe una diferencia significativa.

Fuente: elaboración propia a partir de OECD (2016). PISA 2015 Results (Volume I): Excellence and Equity in Education. OECD Publishing, Paris. doi: 10.1787/19963777

\section{DISCUSIÓN Y PROPUESTAS DE MEJORA}

Como hemos podido observar a lo largo de este trabajo, la población inmigrante se encuentra en una clara desventaja en los contextos educativos de la Unión Europea. En primer lugar, queremos hacer hincapié en la necesidad de que los Estados configuren la distribución del alumnado inmigrante atendiendo a los principios de equidad y de no-discriminación. Tras analizar los datos podemos concluir que el porcentaje de población inmigrante no es un factor condicionante en el rendimiento escolar de este alumnado.

Por otro lado, la segregación escolar sigue muy presente en la gran mayoría de los Estados Miembro. Esta práctica discriminatoria trae consigo un gran abanico de consecuencias negativas para toda la población, no sólo la inmigrante. Por un lado, perpetúa la situación de desventaja social de la población de origen inmigrante, consolidando estereotipos y visiones distorsionadas y xenófobas de estas personas. Estos centros educativos en su relación e impacto con la comunidad convierten a esas zonas geográficas en barrios con un bajo prestigio social. Estas visiones no hacen más que ahondar en una desigualdad estructural y generan cierto rechazo a la población del país de acogida a vivir y llevar a sus hijos a esas escuelas (Martínez-Usarralde, Lloret-Catalá y Céspedes, 2017), dificultando la inclusión de las personas que conviven a los alrededores de esos centros. Por otro lado, la mayoría del alumnado inmigrante está escolarizado en centros de titularidad pública, y en el caso de España, como bien señala Save the Children (2018), los centros concertados están eludiendo sus responsabilidades con el Estado y con la ciudadanía al añadir sobrecostes a sus estudiantes. En vista de las repercusiones que tiene la segregación y la acumulación premeditada del alumnado inmigrante en las escuelas públicas, podría afirmarse que la escuela pública está perdiendo atractivo y devaluando su valor para la sociedad.

El contexto político de la Unión Europea no muestra demasiadas esperanzas para revertir esta situación. En el seno de la Unión Europea emergen cada vez con más fuerza partidos políticos de ultraderecha reticentes a la inmigración, por ejemplo: el Frente Nacional (Francia), Vox (España), el Partido de la Libertad (Austria), HSP (Croacia), Amanecer Dorado (Grecia), Jobbik (Hungría), DF (Dinamarca), UKIP (Reino Unido) o Verdaderos Finlandeses (Finlandia) (Montoya, 2017). Bien es cierto que históricamente este organismo supranacional ha mostrado su preocupación por esta cuestión, como se refleja en: la Directiva 77/486/CEE y las Conclusiones 2009/C 301/07. Sin embargo, su actuación hasta la fecha podría catalogarse de insuficiente en vista de los resultados obtenidos en anteriores investigaciones (Zinovyeva, Felgueroso y Vázquez, 2009; Etxeberría y Elosegui, 2010) y en esta misma. 
Por otro lado, es importante atender a la diferencia que existe entre el alumnado inmigrante de primera generación y de segunda. En esta investigación se ratifica la gran influencia del contexto socioeconómico en el rendimiento académico del alumnado inmigrante (Zinovyeva, Felgueroso y Vázquez, 2009; Escarbajal, Sánchez y Guirao, 2015), pero es preciso ir más allá. Etxeberría (2009) destaca la falta de medios y de recursos de los centros educativos y el profesorado para atender al alumnado de incorporación tardía al sistema educativo, aunque en el informe PISA 2015 tan sólo Estonia, Italia y Luxemburgo tenían un déficit de recursos. Sin embargo, PISA no puede evaluar la eficacia y la eficiencia de estos recursos, pues la mera existencia de ellos no supone que sean los adecuados. Esta observación de Etxeberría (2009) se enmarca perfectamente con la política educativa supranacional de la Unión Europea de mejorar la formación inicial y permanente del profesorado (Valle, 2012). El cuerpo docente se erige como el gran activo social, político, económico y educativo para alcanzar una mayor cohesión social. Por este motivo, es trascendental que los Estados Miembro mejoren y aumenten en sus programas de formación inicial y permanente del profesorado la interculturalidad, la atención a la diversidad, la ciudadanía europea la adquisición de otras lenguas y la promoción de los derechos humanos y de la infancia. También es preciso que se mejore la atención temprana y los programas de acogida para el alumnado de nueva incorporación o de incorporación tardía al sistema educativo.

Sin embargo, si ponemos en diálogo a las teorías explicativas desarrolladas en el marco teórico y los resultados obtenidos, podemos encontrar ciertas relaciones entre ellas, que expliquen la diferencia en el rendimiento académico entre el alumnado inmigrante y nativo en los países con más desigualdad. En primer lugar, como indicábamos anteriormente, estas diferencias se acentúan en los países donde los resultados superan los 500 puntos y, por ende, son más exitosos. La "teoría del déficit" (Rodríguez Izquierdo, 2010) nos permite dar una respuesta a este fenómeno, pues el alumnado inmigrante tiene un bagaje y un nivel educativo previo más bajo que sus nuevos compañeros en el país de acogida, lo que también explicaría por qué se reduce la diferencia entre el alumnado inmigrante de segunda generación y el nativo. No obstante, la "teoría de la discontinuidad cultural" (Rodríguez Izquierdo, 2010) emana como una posible justificación a los datos presentados anteriormente, pues el choque cultural y lingüístico dificulta significativamente el aprendizaje del menor extranjero. Para terminar, la "teoría de la construcción social de la desventaja" (Rodríguez Izquierdo, 2010) enriquece el análisis gracias a su enfoque holístico e integral. Así, hemos podido identificar cómo los países donde hay más desigualdad en el rendimiento académico se sitúan al norte de Europa, donde sus ciudadanos poseen un fuerte poder adquisitivo. De este modo, a lo largo de la investigación hemos encontrado evidencias de que el nivel sociocultural y económico tiene una influencia más negativa en el alumnado inmigrante, como hemos podido observar en las tablas de resultados en las diferentes competencias y en el porcentaje de alumnado resiliente, pues para ellos, estos factores se convierten en gélidas montañas escarpadas imposibles de escalar.

Para terminar, la última reflexión sobre la que habría que incidir, es en erradicar la idea de que la calidad de un sistema educativo se mide con el rendimiento académico de sus alumnos en PISA. Lejos de entrar en el debate sobre la influencia, los beneficios o los efectos negativos de incorporar este tipo de pruebas de evaluación a los sistemas educativos, sí es oportuno apuntar brevemente que el uso de los datos que estas pruebas aportan se hace de forma sesgada e intencionada por los medios de comunicación, los partidos políticos y los Estados (Fernández-González, 2015; SolanoFlores y Milbourn, 2016). Si adoptamos una visión reduccionista y simplista del análisis de los datos que ofrece PISA, con cierta negligencia defenderemos el gran valor pedagógico de países con excelentes resultados académicos en el informe, e incluso los situaremos como referentes mundiales. Por el contrario, se hace imprescindible adoptar una visión crítica de los datos que nos 
ofrece y emplearlos como un instrumento de diagnóstico, evaluación y mejora de la desigualdad de oportunidades que padecen los sectores más vulnerables en la Unión Europea. En definitiva, el referente y el centro de la acción educativa que debe orientar la política educativa supranacional de la Unión Europea es el interés superior del menor y los derechos humanos (ONU, 1989), pues un sistema educativo que no garantice la igualdad de oportunidades y la plena inclusión de las personas más vulnerables no es un sistema de calidad. 


\section{REFERENCIAS}

ACNUR (2018). Emergencia en Siria. Recuperado de_http://www.acnur.org/emergencia-ensiria.html

Calero, J., Choi, Á. y Waisgrais, S. (2009). Determinantes del rendimiento educativo del alumnado de origen nacional e inmigrante en PISA-2006. Cuadernos económicos de ICE, 78, 281-312.

Comisión Europea (2018a). Estadísticas de asilo. Recuperado de https://ec.europa.eu/eurostat/statistics-

explained/index.php?title=Asylum_statistics/es\&oldid=409325\#Edad_y_g.C3.A9nero_d e_los_nuevos_solicitantes

Comisión Europea (2018b). Estadísticas de migración y población migrante. Recuperado de

https://ec.europa.eu/eurostat/statisticsexplained/index.php?title=Migration and migrant population statistics/es\#Poblaci.C3. B3n migrante: en la UE viven casi 22 millones de ciudadanos de terceros pa.C3.A Dses

Conclusiones del Consejo de 26 de noviembre de 29 de 2009 sobre la educación de los niños procedentes de la migración (2009/C 301/07). Diario oficial, serie C, número 301/5 del 11 de diciembre de 2009.

Cordero Ferrera, J. M., Crespo Cebada, E. y Pedraja Chaparro, F. (2013). Rendimiento educativo y determinantes según PISA: Una revisión de la literatura en España. Revista de Educación, 362. doi: 10.4438/1988-592X-RE-2011-362-161

Directiva del Consejo de 25 de julio de 1977 relativa a la escolarización de los hijos de los trabajadores migrantes (77/486/CEE). Diario oficial, serie L número 199/32, de 6 de agosto de 1977.

Enkvist, I. (2010). El éxito educativo finlandés. Bordón. Revista de pedagogía, 62(3), 49-67.

Escarbajal Frutos, A., Sánchez, M. y Guirao, I. (2015). Factores determinantes del rendimiento académico de alumnos hijos de inmigrantes y autóctonos de origen extranjero en contextos de exclusión social. In Revista sobre la infancia y la adolescencia, 9, 31-46. doi: $10.4995 /$ reinad.2015.3772

Etxeberría, F. y Elosegui, K. (2010). Integración del alumnado inmigrante: obstáculos y propuestas. Revista Española de Educación Comparada, 16, 235-263.

Etxeberría, F., Karrera, I. y Murua, H. (2009). Competencias interculturales del profesorado con alumnado inmigrante en el País Vasco. Revista Electrónica Interuniversitaria de Formación del Profesorado, 13, 4, 79-94.

Fernández-González, N. (2015). PISA como instrumento de legitimación de la reforma de la LOMCE. Bordón, 67, 1, 165-178. doi: 10.13042/Bordon.2015.67111

García Vargas, J. (2015). El efecto Pigmalión y su efecto transformador a través de las expectativas. Perspectivas docentes, 57, 40-43.

Gripenberg, M. y Lizarte Simón, E. J. (2012). El sistema educativo de Finlandia y su éxito en la prueba PISA. Journal for Educators, Teachers and Trainers, 3, 14-24.

Martínez-Usarralde, M. J., Lloret-Catalá, M. C. y Céspedes Rico, M. (2017). Lo que hacen las mejores escuelas integradoras de alumnado inmigrante: indicadores de buenas prácticas. Pedagogía social: revista interuniversitaria, 29, 41-54.

Monarca, H. (2012). La influencia de los sistemas nacionales de evaluación en el desarrollo del currículo. Perfiles educativos, 34(135), 164-176.

Montoya García, J. (2017). Ideologías excluyentes en el siglo XXI. Austria y auge de la extrema derecha en Europa. Pensamiento al margen. Revista digital, 6, 55-75.

OECD (2016). PISA 2015 Results (Volume I): Excellence and Equity in Education. OECD Publishing, Paris. doi: 10.1787/19963777 
ONU (1989). Convención sobre los Derechos del Niño. Recuperado desde http://www.un.org/es/events/childrenday/pdf/derechos.pdf

Pérez, A. I. y Soto, E. (2011) Luces y sombras de PISA. Sentido educativo de las evaluaciones externas. Cultura y Educacion, 23(2), 171-182. doi: 10.1174/113564011795944758

Regueiro, B., Suárez Fernández, N., Estévez, I., Rodríguez, S., Piñeiro, I. y Valle, A. (2018). Deberes escolares y rendimiento académico: un estudio comparativo entre el alumnado inmigrante y nativo. Revista de Psicología y Educación, 13, 2, 92-98. doi:10.23923/rpye2018.01.160

Rodríguez Izquierdo, R. M. (2010). Éxito académico de los estudiantes inmigrantes. Factores de riesgo y de protección. Educación XXI, 13, 1, 101-123.

Sahlberg, P. (2015). Un sistema escolar modelo. Finlandia demuestra que la equidad y la excelencia pueden coexistir en la educación. Pensamiento Educativo. Revista de Investigación Educacional Latinoamericana, 52(1), 136-145.

Save the Children (2018). Mézclate conmigo. De la segregación socieconómica a la educación inclusiva. https://www.savethechildren.es/sites/default/files/imce/docs/mezclate conmigo.pdf

Solano-Flores, G. y Milbourn, T. (2016). Capacidad evaluativa, validez cultural y validez consecuencial en PISA. RELIEVE, 22, 1,1-17. doi: 10.7203/relieve.22.1.8281

Torrado Martín-Palomino, E. (2012). Las migraciones de menores no acompañados desde una perspectiva de género. Dilemata, 10, 65-84.

UNICEF (2017). Harrowing Journeys. Children and youth on the move across the Mediterranean Sea, at risk of trafficking and exploitation. Recuperado de https://www.unicef.org/publications/files/Harrowing_Journeys Children and youth o $\mathrm{n}$ the move across the Mediterranean.pdf

Valle, J. M. (2012). La formación inicial del profesorado según la Unión Europea. Nueva Revista, 141, 32-50.

Zinovyeva, N., Felgueroso, F. y Vázquez, P. (2009). Inmigración y resultados educativos en España. Comunicación presentada en Efectos económicos de la Inmigración en España. Jornadas sobre inmigración. I Informe FEDEA, Madrid, 139-178. 


\section{SOBRE EL AUTOR}

\section{Adrián Neubauer Esteban}

Graduado en magisterio de Educación Infantil (UCJC) y Educación Primaria (UAM). Máster en Calidad y Mejora de la Educación (UAM). Experiencia laboral: Maestro de Educación Primaria en la Comunidad de Madrid. Actualmente contratado predoctoral FPI en el Departamento de Pedagogía, de la Facultad de Formación de Profesorado y Educación en la Universidad Autónoma de Madrid. Actividad investigadora: Miembro del Grupo de Investigación sobre Políticas Educativas Supranacionales (GIPES-UAM). Lineas de investigación: derechos humanos, derechos de la infancia, derecho a la educación y política educativa.

Información de contacto: adrian.neubauer@uam.es 